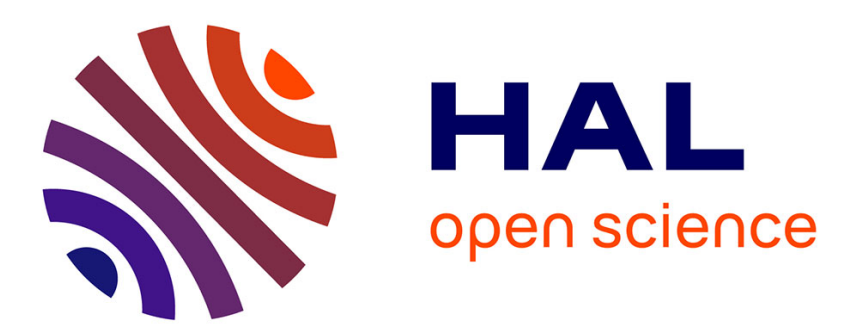

\title{
Field Grading Composites Tailored by Electrophoresis -Part 1: Principle and Permittivity Gradient in Uniform Electric Field
}

\author{
Sombel Diaham, Z. Valdez-Nava, L. Lévêque, T. T Le, Lionel Laudebat, \\ Thierry Lebey
}

\section{To cite this version:}

Sombel Diaham, Z. Valdez-Nava, L. Lévêque, T. T Le, Lionel Laudebat, et al.. Field Grading Composites Tailored by Electrophoresis -Part 1: Principle and Permittivity Gradient in Uniform Electric Field. IEEE Transactions on Dielectrics and Electrical Insulation, 2021, 28 (2), pp.333-340. 10.1109/TDEI.2020.009030 . hal-03266259

\section{HAL Id: hal-03266259 \\ https://hal.science/hal-03266259}

Submitted on 21 Jun 2021

HAL is a multi-disciplinary open access archive for the deposit and dissemination of scientific research documents, whether they are published or not. The documents may come from teaching and research institutions in France or abroad, or from public or private research centers.
L'archive ouverte pluridisciplinaire HAL, est destinée au dépôt et à la diffusion de documents scientifiques de niveau recherche, publiés ou non, émanant des établissements d'enseignement et de recherche français ou étrangers, des laboratoires publics ou privés. 


\title{
Field Grading Composites Tailored by Electrophoresis - Part 1: Principle and Permittivity Gradient in Uniform Electric Field
}

\author{
S. Diaham ${ }^{1}$, Z. Valdez-Nava ${ }^{1}$, L. Lévêque ${ }^{1}$, T. T. Le ${ }^{1}$, L. Laudebat ${ }^{1,2}$ and T. Lebey ${ }^{1}$ \\ ${ }^{1}$ LAPLACE, Université de Toulouse, CNRS, INPT, UPS, Toulouse, France \\ ${ }^{2}$ Université de Toulouse, INU Champollion, place de Verdun F-81012 Albi, France.
}

\begin{abstract}
A series of three articles present an innovative way to build advanced functionally graded materials (FGM) based on polymer/ceramic composites tailored by electrophoresis from the process principle to their field grading application in power electronics. In this Part 1 , the process is presented and relies on applying a DC voltage on liquid composite compound before curing in order to 'freeze' the particles where they have been accumulated. To exemplify this principle, FGM composites involving an epoxy resin with $\mathrm{SrTiO}_{3}$ high- $k$ particles with a permittivity gradient are presented. The methodology to build and characterize them is carried out. An accumulated particle region is observed at the high voltage electrode while the depleted bulk region remains unmodified. This accumulated particle layer both increases in thickness and in filler densification with increasing the field and/or time allowing tuning its permittivity. This work paves the way to the development of more robust electronic systems where the electrical fringe field in critical regions can be mitigated.
\end{abstract}

Index Terms - functionally graded materials (FGM), composite, electrophoresis, insulation, encapsulation, high voltage, field grading, power electronics modules

\section{INTRODUCTION}

WITH the rapid and recent progresses in the development of advanced electronics systems and active devices, the polymer materials often used for the packaging of such systems or as active materials are facing physical limits thermally, electrically and mechanically during aging under combined multi-stress conditions [1],[2]. This is the case for polymeric dielectrics and insulators, organic joints or adhesives and printed circuit board substrates and encapsulation. Polymers such as epoxy resin, polyvinylidene fluoride, polypropylene, polyimide, silicone gel, silicone rubber are usually under consideration in these applications.

Composite materials, based on the mixing of both a polymer matrix filled with inorganic particles (ceramic or metallic), appear in recent years more and more useful in electronic applications to mitigate the physical constraints and to push up the polymer limits (e.g. enhanced intrinsic performances, higher stability, increase in the lifetime) $[3,4]$. So far, composites enable a fair tradeoff in the material properties trying to improve the weakest one without being, as much as possible, detrimental for the other ones. Polymerbased composites should become ubiquitous to enhance electrical, thermal and mechanical performances of energy conversion systems in many electronic application fields such as high voltage engineering [5-7], power electronics [8, 9], microelectronics [10], capacitor technologies [11, 12], batteries [13].

However, in most of these applications, one can observe that the physical constraints to which these materials (composites or not) are subjected are mostly nonhomogeneous with strong anisotropic characteristics: e.g. local electric field reinforcements at metal track edges, unidirectional heat extraction, in-plane CTE mismatching between materials, etc. To overcome these issues, the community working on composite materials has always had, so far, a standard functionalization approach. Particularly, researchers have developed a lot of composites with homogeneous distribution of the inorganic particles within polymers, thus modifying one (or more) property isotropically in the entire bulk. From the simple observation that the constraints in electronic systems are mainly localized, it should be more relevant to design the composite properties in an anisotropic way or in the form of a gradient by structuring them locally. 

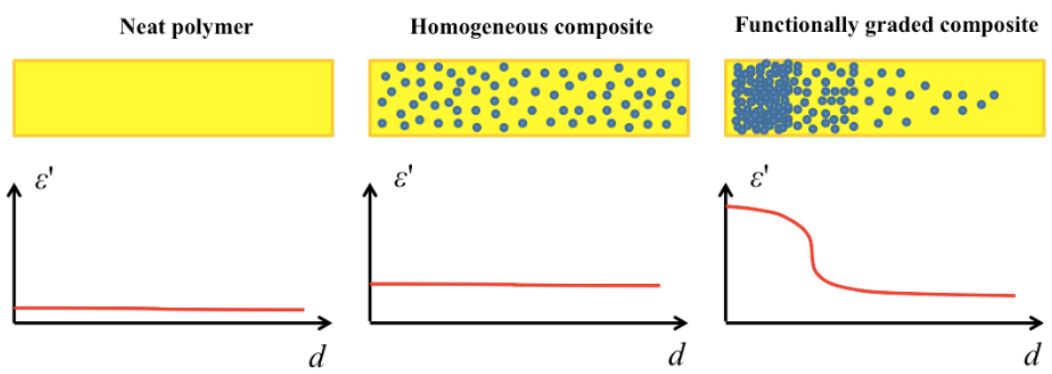

Figure 1. Impact of high permittivity particles arrangement in a polymer composite on the permittivity gradient along a distance: (a) neat polymer, (b) particle homogenous distribution, (c) particle gradient distribution (FGM with gradient).

Functionally graded materials (FGM) are a class of materials that are tailored to have a spatial evolution of at least one property in one direction thanks to a gradual evolution in their structuration. They can be processed either by multilayer deposition (i.e. each layer owns specific properties) or in the case of composites by creating a particle spatial distribution thus offering a property gradient within an initial isotropic matrix [14]. In this latter case, a certain gradient in the electrical conductivity, wear resistance or other mechanical properties can be created in order to smooth or adapt the constraints between materials. Figure 1 exemplifies the principle of the effect of high permittivity particle spatial distribution in a matrix on the overall composite permittivity in the direction of the gradient. Several studies have already reported that such FGM polymer-based composites with particle distribution gradient, processed using centrifugation, exhibit properties gradient in the direction of the applied centrifugal force. Those materials found applications in high voltage engineering for electric field grading $[15,16]$, as well as in space-craft applications by tuning the elastic modulus [17 18]. However, for more integrated electronic systems (as power modules), such centrifugation process is no longer suitable to tailor locally a property gradient exactly where the electric field constraints are the most severe. A well-known and potential suitable process to accumulate particles, but never used for such applications, could be the electrophoresis.

Electrophoresis is a physical process, which consists in displacing particles within a liquid medium under the application of an electrostatic force (i.e. the Coulomb force) on the compound [19]. The trapping of electric charges at the interface between the particles and the liquid enables the particles to move towards the electrode of opposite potential. Even if electrophoresis has been extensively used to develop co-ceramic FGM composites [20], it has recently been firstly used and reported for polymer-based composites and in that purpose by our LAPLACE research group [21-23]. Since then, another study has reported also the interest of similar epoxy/BaTiO 3 FGM for $\mathrm{HV}$ application [7].

In this paper, the authors propose an original way to tailor by electrophoresis advanced FGM polymer-based composites with properties gradient [21]. Such FGM are obtained by applying a DC voltage on the liquid composite compound before curing it in order to 'freeze' the particles exactly where they have been accumulated. Such process is very versatile and adaptable from small size to largedimensions electronic and electrical systems by directly using the electrodes of the systems themselves. A particular example of a FGM composite with a permittivity gradient (epoxy/$/ \mathrm{SrTiO}_{3}$ ) is developed all along this series of papers for explaining the process, the characterization methodologies and its application for the encapsulation of power modules.

\section{EXPERIMENTAL}

\subsection{MATERIALS}

In this study, the polymer used as the host matrix is a diglycidyl ether of bisphenol $\mathrm{F}$ (DGEBF) thermosetting epoxy resin (cf. Figure 2). It is crosslinked using an aminetype hardener with a ratio of 10:1 (resin:hardener). The viscosity of the epoxy resin and the harder mixture is 3000 $\mathrm{mPa} . \mathrm{s}$ at room temperature and its density after curing is 1.23 $\mathrm{g} / \mathrm{cm}^{3}$ and a large and high continuous operating temperature range ( -55 to $230{ }^{\circ} \mathrm{C}$ ) compatible with standard power electronics applications $\left(125^{\circ} \mathrm{C}\right)$.

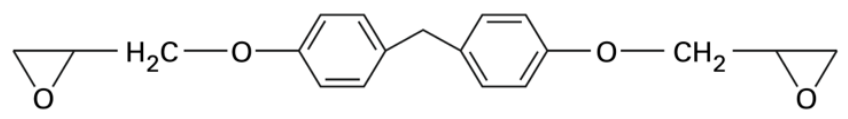

Figure 2. Chemical structure of DGEBF epoxy.

The high permittivity fillers used here are strontium titanate $\left(\mathrm{SrTiO}_{3}\right)$ particles with a density of $5.11 \mathrm{~g} / \mathrm{cm}^{3}$ and a BET specific surface of $1.83 \mathrm{~m}^{2} / \mathrm{g}$. $\mathrm{SrTiO}_{3}$ has the twofold advantages of a negative Curie temperature not interfering with the application range, and of a higher dielectric strength $\left(35 \mathrm{kV} / \mathrm{mm}\right.$ ) compared to $\mathrm{BaTiO}_{3}$ (approx. $5 \mathrm{kV} / \mathrm{mm}$ ).

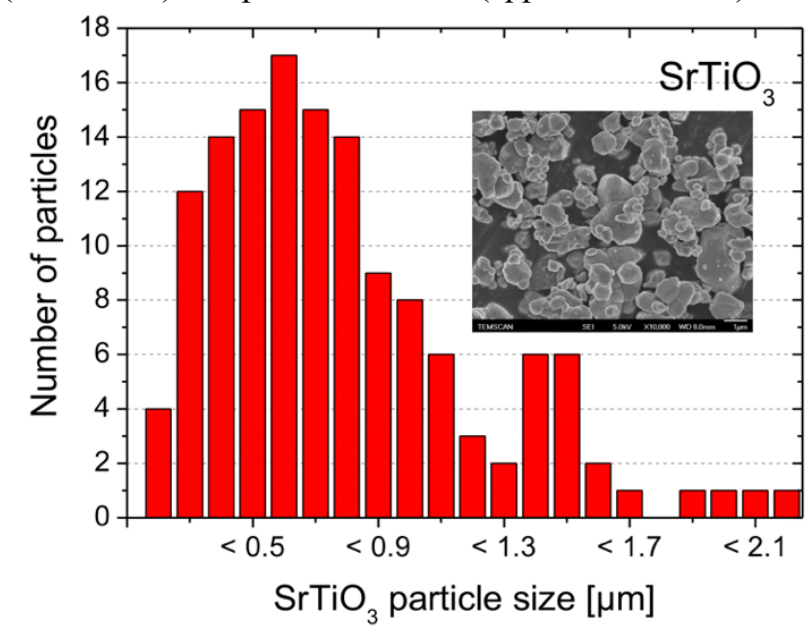

Figure 3. $\mathrm{SrTiO}_{3}$ particle size distribution. The inset shows a SEM image of the particles (the scale bar is $1 \mu \mathrm{m}$ ). 
$\mathrm{SrTiO}_{3}$ particles used are polyhedral-shaped and present an average diameter of $600 \mathrm{~nm}$, as shown in Figure 3.

\subsection{ELECTROPHORESIS PROCESS}

A first step in the composite mixture elaboration process was achieved by a direct blending process of the $\mathrm{SrTiO}_{3}$ particles into the liquid epoxy resin containing the hardener. The liquid composite mixture, with an initial filler content of 10 vol\%, was homogeneously dispersed using a planetary mixer at $3000 \mathrm{rpm}$ for 8 minutes. The mixture was then degassed in primary vacuum $(<1.5 \mathrm{kPa})$.

For real-time optical microscopy observations of the $\mathrm{SrTiO}_{3}$ particle movements in the resin, the mixture was poured between two $\mathrm{Cu}$ parallel plates $(1 \mathrm{~mm} \times 5 \mathrm{~mm} \times 20 \mathrm{~mm})$ separated by $1 \mathrm{~mm}$-thick glass spacers and glued on a glass slide (see Figures 4a and 4b). DC electric fields were applied on the $\mathrm{Cu}$ electrodes using a voltage source Keithley SM 2410 ranging $125 \mathrm{~V}$ up to $500 \mathrm{~V}$ and for durations from 1 to 60 minutes (see Figure 4c). After switching the voltage off, the sample cell was put into an oven in order to cure the FGM epoxy/SrTiO 3 compound and to 'freeze' the particles where they have been accumulated. The samples were cured at 150 ${ }^{\circ} \mathrm{C}$ for $20 \mathrm{~min}$ in air for crosslinking the epoxy resin (see Figure 4d). For making larger FGM samples suitable for dielectric characterizations, the same process was achieved in stainless steel molds of larger area $\left(7 \times 7 \mathrm{~cm}^{2}\right)$. In this case, the DC voltage was applied between the two sides of the mold separated by an insulating joint spacer of $1 \mathrm{~mm}$-thick. The same curing protocol was used.

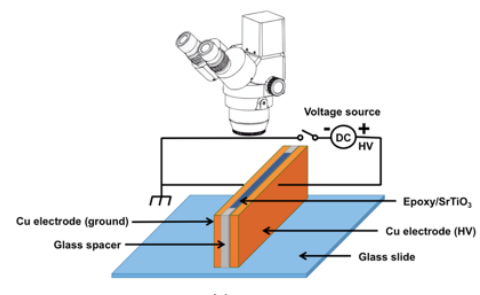

(a)

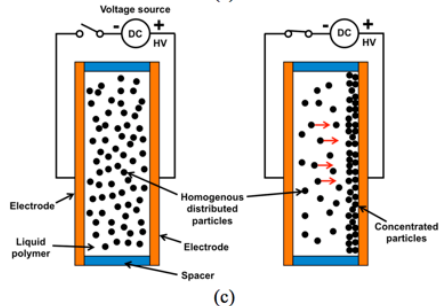

(c)

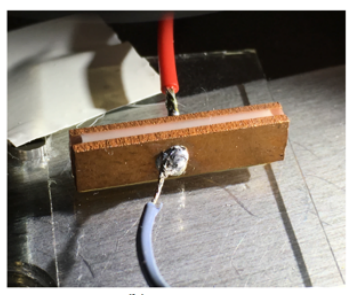

(b)

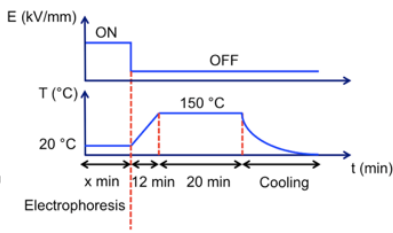

(d)
Figure 4. Principle of the electrophoresis process under DC voltage applied to a liquid polymer/ceramic compound and curing process.

\subsection{MEASUREMENTS}

The FGM accumulated layer thickness and the particle distribution were characterized using a field-emission gun scanning electron microscope (FEG-SEM JEOL JSM$7800 \mathrm{~F}$ ) coupled to energy dispersive spectroscopy (EDS), which enables to qualitatively and quantitatively identify the elemental distribution of $\mathrm{Sr}$ and $\mathrm{Ti}$ atoms from the $\mathrm{SrTiO}_{3}$ particles. The weight fractions of $\mathrm{SrTiO}_{3}$ particles present in each region (accumulated layer and depleted zone) of the FGM epoxy/SrTiO 3 composites were determined by thermogravimetric analysis (TGA) using a Perkin-Elmer Instruments Diamond analyzer. The measurements were achieved on around $10 \mathrm{mg}$ of composite matter picked from each region of the FGM after polishing mechanically the other one. The measurements were performed with a temperature rising of $10{ }^{\circ} \mathrm{C} / \mathrm{min}$ up to $800{ }^{\circ} \mathrm{C}$ in air atmosphere in order to totally degrade the epoxy matrix and to only conserve the particle fraction. The $\mathrm{SrTiO}_{3}$ volume fraction was then calculated using the particle and epoxy densities. The dielectric permittivity $\left(\varepsilon^{\prime}\right)$ has been measured by broadband dielectric spectroscopy using a Novocontrol Alpha-A spectrometer with a ZGS testing cell. Measurements have been performed at room temperature in a frequency range from $100 \mathrm{mHz}$ to $1 \mathrm{MHz}$. Each region of the FGM was characterized after polishing the other one and deposited gold sputtered electrodes.

\section{RESULTS AND DISCUSSION}

\subsection{PARTICLE GRADIENT FORMATION}

During the experiments, it was possible to highlight that the dynamics of the particle concentration towards the higher potential electrode was strongly influenced by many parameters such as the field magnitude, time, the field polarity, the initial filler content, the particle size, the particle permittivity/ conductivity, the matrix permittivity/ conductivity, the zeta potential around the particles and the viscosity of the resin.

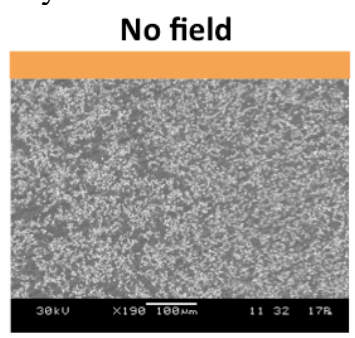

$5 \mathrm{~min}$

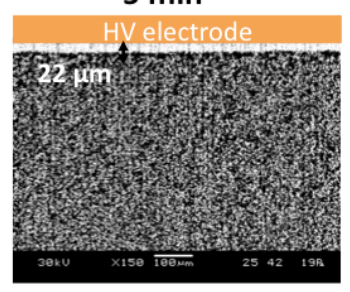

$30 \mathrm{~min}$

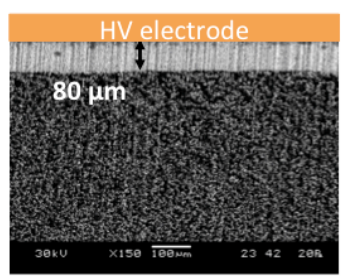

Electric field $=125 \mathrm{~V} / \mathrm{mm}$

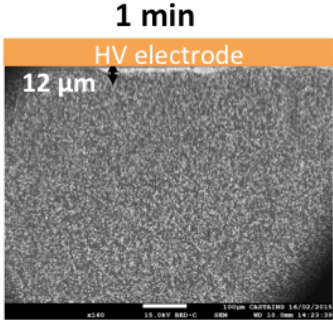

$15 \mathrm{~min}$

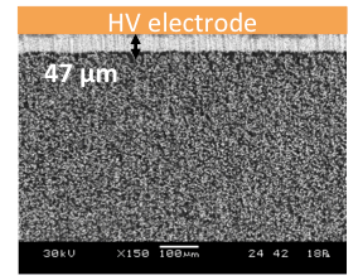

$60 \mathrm{~min}$

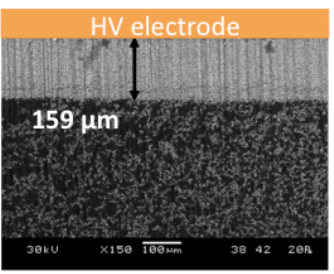

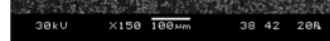

Figure 5. Cross-section SEM images of the epoxy/SrTiO 3 composites (initial content: $10 \mathrm{vol} \%$ ) structured by electrophoresis in a DC electric field of $125 \mathrm{~V} / \mathrm{mm}$ for different times. 
Even though the complete study that deals with the influence of all those parameters was carried out [23], only the influence of the electric field magnitude and time is detailed in the present report.

Figure 5 presents cross-section SEM images of the FGM epoxy/$/ \mathrm{SrTiO}_{3}$ composites structured by electrophoresis under a DC electric field of $125 \mathrm{~V} / \mathrm{mm}$ and for different times. under DC field, the formation of a $\mathrm{SrTiO}_{3}$ particle layer collected at the HV electrode is observed. This layer progressively increases in thickness with time starting from few microns up to more than $100 \mu \mathrm{m}$ after $1 \mathrm{~h}$ showing a first possibility to tune this layer in the desired dimension. This layer exhibits conformal geometrical aspects and covers the entire electrode set at HV. The rest of the FGM composite (i.e. the depleted region) still contains $\mathrm{SrTiO}_{3}$ particles homogeneously dispersed in the epoxy bulk.

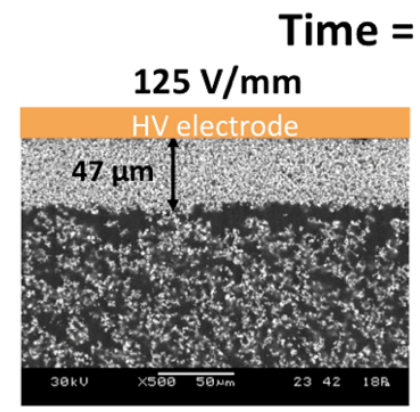

$375 \mathrm{~V} / \mathrm{mm}$
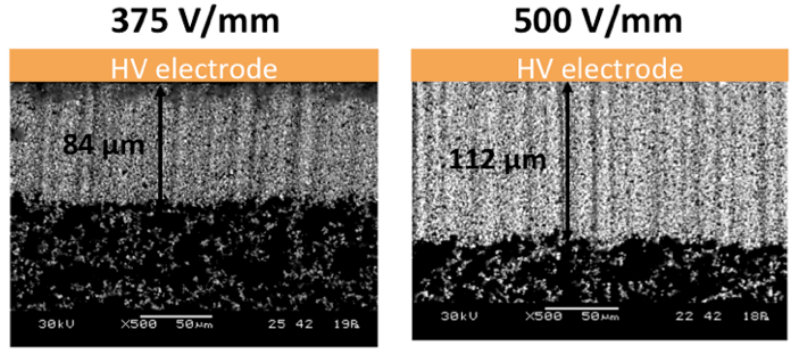

Figure 6. Cross-section SEM images of the epoxy/ $/ \mathrm{SrTiO}_{3}$ composites (initial content: $10 \mathrm{vol} \%$ ) structured by electrophoresis at different DC electric fields for 15 minutes.

Figure 6 shows cross-section SEM images of the FGM epoxy/SrTiO 3 composites after the structuration during 15 minutes under different DC electric fields. In this case, one can observe the effect of increasing the electric field on the dynamics of the particle layer deposition. For such a short duration of the field application, the conformity of the layer remains homogeneous. Moreover, as the layer thickness increases with the field, the depleted region seems to present a clear decrease in its filler density. Such higher depletions of the $\mathrm{SrTiO}_{3}$ particles from the bulk of the composites could be related either to a more important densification in the particle layer with the field increasing or just to its thickness increase at a constant content inside. This will be emphasized in the following parts.

\subsection{INHOMOGENEITY ISSUES AT LONG TIMES}

Figure 7 shows cross-section SEM images of the FGM epoxy/ $\mathrm{SrTiO}_{3}$ composites after the structuration during 60 minutes under different DC electric fields. It is worth to note that combining both high electric fields and long application times is detrimental in terms of the particle layer homogeneous conformation.

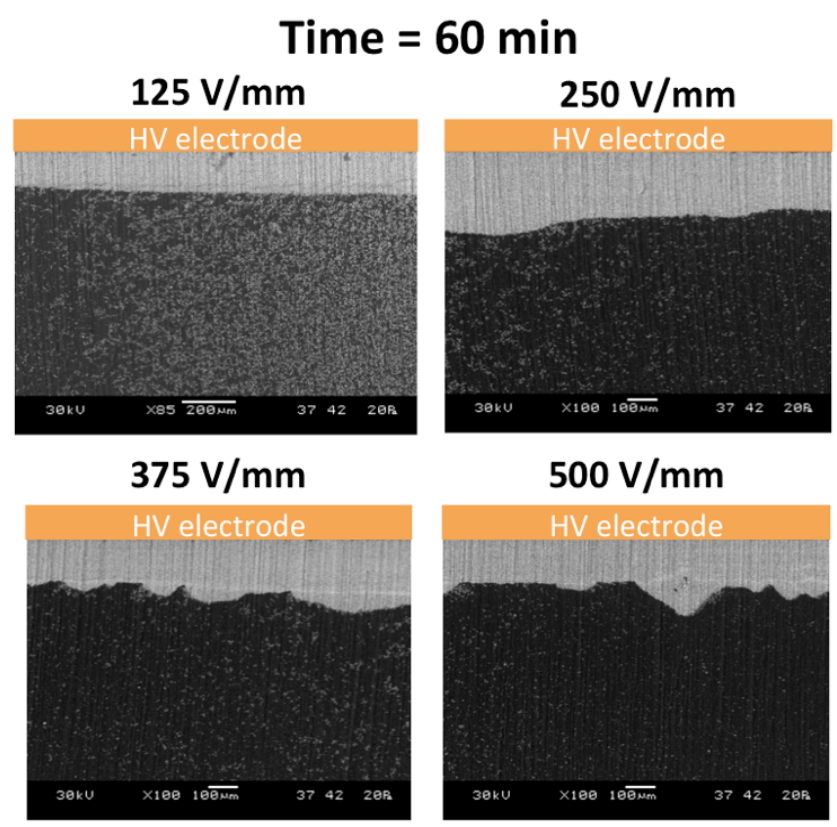

Figure 7. Cross-section SEM images of the epoxy $/ \mathrm{SrTiO}^{3}$ composites (initial content: $10 \mathrm{vol} \%$ ) structured by electrophoresis at different DC electric fields for 60 minutes.

Actually, after $1 \mathrm{~h}$ of electrophoresis, one can clearly observe from $250 \mathrm{~V} / \mathrm{mm}$ and above that the FGM epoxy/SrTiO 3 composites exhibit non-uniformities in the growth of the particle layers. These last ones present large variations of their thickness along the HV electrodes. Such thickness inhomogeneities could be explained by the progressive occurrence during the electrophoresis process of localized regions of higher particle concentrations leading to high permittivity/conductivity regions. Thus, these particle aggregations in the layer could act as higher electric field regions that locally enhance the field viewed by the other particles in movement. Consequently, they could be preferentially attracted by these higher electric field areas, thus building peaks of particles within the accumulated layer. Moreover, such a combination between the high field and its long application time leads at the end to an almost complete depletion of particles from the rest of the composite (e.g. at $500 \mathrm{~V} / \mathrm{mm}$ ). Such conditions structure the FGM composite in the way of a high permittivity layer and a bulk of lower one probably similar to that of the epoxy matrix.

For building FGM composites with a less abrupt permittivity difference between the two regions (i.e. accumulated and depleted), a strategy would be to work under lower applied electric fields and to use the application time as a thickness tuning parameter.

\subsection{PARTICLE LAYER THICKNESS}

Figure 8 presents a summary of the thickness changes in the accumulated particle layer of the FGM epoxy/SrTiO composites versus the application time and for different applied electric fields. 


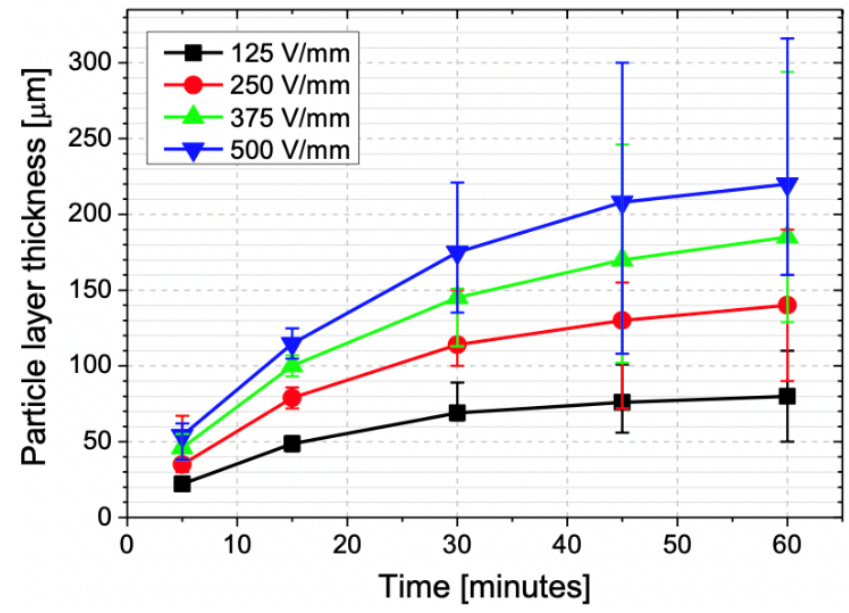

Figure 8. Thickness changes of the accumulated particle layer versus time for different applied electric fields.

The thickness of the accumulated particle layer increases asymptotically with time at a given field. Moreover, the initial increase (initial slope) appears more pronounced when the applied field is higher. As explained previously, the thickness inhomogeneity leads to larger error bars (dispersion) around the average thickness of the layer for the longest times and the highest electric fields. The present results confirm the kinetics tendency of what is typically observed in electrophoretic deposition following the deposited weight of the accumulated layer [24]. The SarkarNicholson kinetics Equation [25] for the deposited weight, can be easily modified by a similar equation describing the increase in the accumulated particle layer thickness $(e)$ and expressed as an exponential function of time:

$e(t)=e_{\infty}(1-\exp (-k t))$

where $e_{\infty}$ is the maximum particle layer thickness reachable at infinite time and $k$ a parameter describing the accumulation kinetics. Table 1 summarizes for each electrical field the maximum thickness reachable and the kinetics parameter of the layer deposition.

Table 1. Parameters describing the kinetics of the particle layer accumulation extracted from eq. (1) for the different applied fields.

\begin{tabular}{ccc}
\hline Applied field $(\mathrm{V} / \mathrm{mm})$ & $e_{\infty}(\mu \mathrm{m})$ & $k\left(\mathrm{~s}^{-1}\right)$ \\
\hline 125 & 82.4 & $5.80 \times 10^{-2}$ \\
250 & 145.0 & $5.08 \times 10^{-2}$ \\
375 & 188.5 & $4.76 \times 10^{-2}$ \\
500 & 249.9 & $3.72 \times 10^{-2}$ \\
\hline
\end{tabular}

\subsection{PARTICLE DISTRIBUTION}

In order to confirm the profile of the particle distribution in the FGM, EDS measurements were achieved on the SEM images of the samples. Figure 9 presents an example of the EDS profiles of $\mathrm{Sr}$ and Ti elements along the particle gradient for an epoxy/SrTiO 3 FGM composite processed at 500 $\mathrm{V} / \mathrm{mm}$ for 15 minutes.

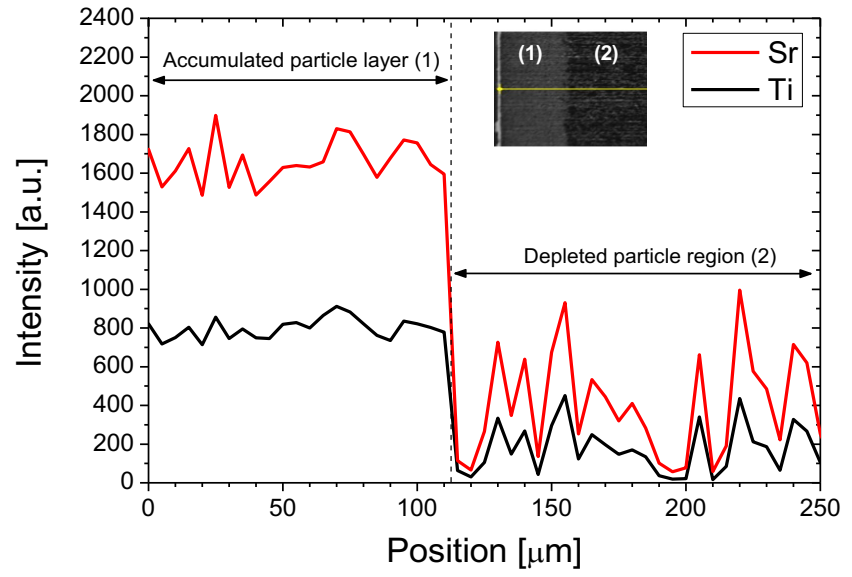

Figure 9. EDS profiles of $\mathrm{Sr}$ and $\mathrm{Ti}$ elements along the particle gradient for an epoxy/SrTiO3 FGM composite processed at $500 \mathrm{~V} / \mathrm{mm}$ for 15 minutes. Position ' 0 ' corresponds to the HV electrode location. The measurement is only given along the first $250 \mu \mathrm{m}$ of the sample. The inset corresponds to the SEM image where (1) is the accumulated particle layer and (2) is the depleted region.

As expected, the intensity detected for both $\mathrm{Sr}$ and $\mathrm{Ti}$ atoms are larger in the accumulated particle layer than those in the depleted region of the FGM. This is in good agreement with the concentration of $\mathrm{SrTiO}_{3}$ particles at the $\mathrm{HV}$ electrode. Moreover, it is worth to note that the elementary profiles in the accumulated layer is constant all along the thickness emphasizing a homogeneous particle concentration of the particle in this layer. The abrupt decrease in the $\mathrm{Sr}$ and $\mathrm{Ti}$ atom intensities detected when entering into the depleted region confirms a well-marked transition between the two regions of the FGM composite, which should have different dielectric properties. In the depleted particle region, apparently the noise level of the EDS signal appears as higher, this is due to the presence of $\mathrm{SrTiO}_{3}$ particles or aggregates within the resin that increase very locally the X-ray emission. However, in the present case, the results highlight the FGM composites structuration exhibiting two regions with different particle concentrations (i.e. high and low).

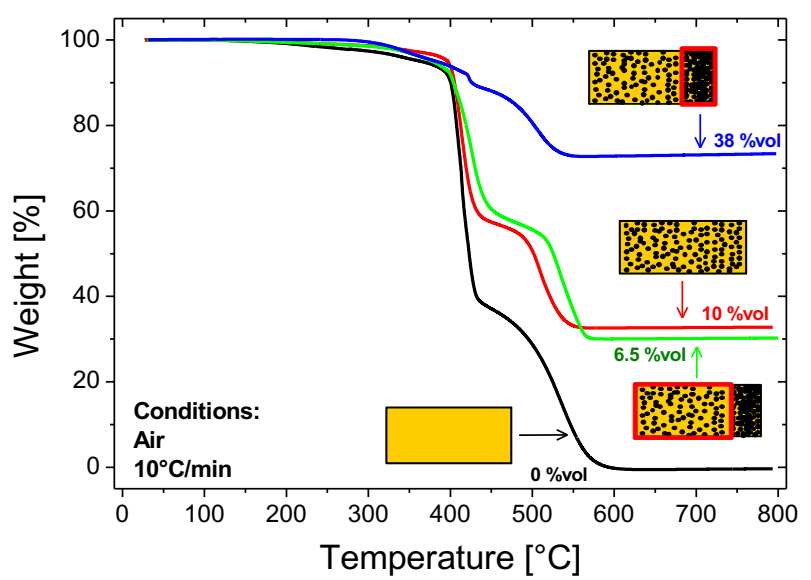

Figure 10. TGA thermographs in the two regions (accumulated and depleted) of an epoxy/SrTiO 3 FGM composite processed at $250 \mathrm{~V} / \mathrm{mm}$ for 60 minutes. Comparison with the homogeneous epoxy/SrTiO ${ }_{3}$ composite (10 vol\%) and the neat epoxy. 
To characterize precisely the $\mathrm{SrTiO}_{3}$ filler content in each region, weight loss measurements were performed on samples after polishing the other one. Figure 10 shows TGA thermographs up to $800{ }^{\circ} \mathrm{C}$ in air for the two regions (accumulated and depleted) of an epoxy/SrTiO 3 FGM composite (initially $10 \mathrm{vol} \%$ ) processed at $250 \mathrm{~V} / \mathrm{mm}$ for 60 minutes. From the residual weight of $\mathrm{SrTiO}_{3}$ particles obtained above $600{ }^{\circ} \mathrm{C}$, after a complete degradation by oxidation of the epoxy matrix, it is possible to calculate the equivalent volume filler content. This technique seems to be efficient, since we confirmed that for an homogeneous epoxy $/ \mathrm{SrTiO}_{3}$ composite prepared with $10 \mathrm{vol} \%$ of particles the TGA analysis yielded also $10 \mathrm{vol} \%$. Thus, for the FGM depleted region, it was obtained that the filler content decreased slightly of only few vol\%. The concentration of this small content coming from a large volume and moving into a smaller one (i.e. the accumulated layer) largely increases the $\mathrm{SrTiO}_{3}$ volume content up to $38 \mathrm{vol} \%$.

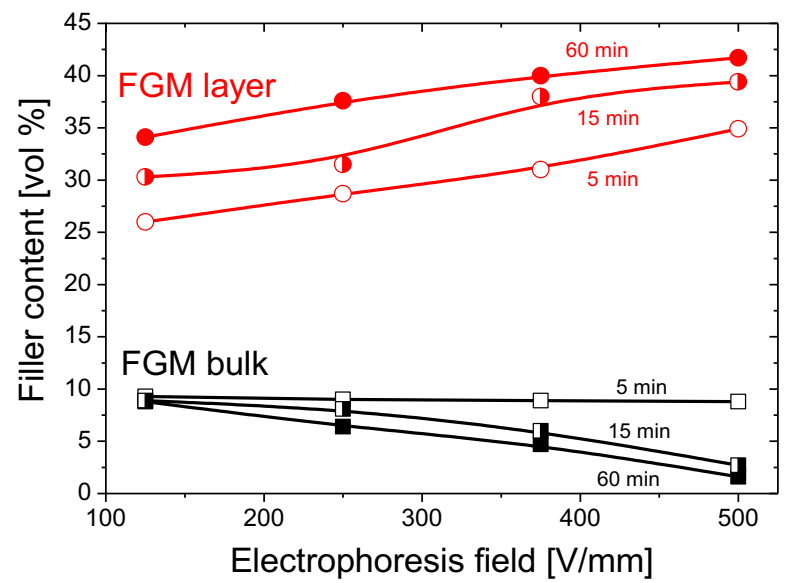

Figure 11. Filler content in the two regions (accumulated and depleted) of various epoxy $/ \mathrm{SrTiO}_{3} \mathrm{FGM}$ composites versus the electrophoresis field and for different times.

Figure 11 presents the filler content changes in each region (layer and bulk) of the FGM as a function of the electrophoretic field and for different times. One can observe that the filler content in the particle layer is increasing with the electrophoresis field and process time while it is decreasing simultaneously in the bulk region. This confirms the observations of SEM images. Moreover, this confirms that not only the thickness increases but also the FGM layer density increases through an enhancement of the particle packing.

\subsection{PERMITTIVITY}

Figure 12 shows the dielectric permittivity and losses for the two regions (accumulated and depleted) of an epoxy $/ \mathrm{SrTiO}_{3} \mathrm{FGM}$ composite (initially $10 \mathrm{vol} \%$ ) processed at $250 \mathrm{~V} / \mathrm{mm}$ for 60 minutes. While the neat epoxy and the homogeneous epoxy/SrTiO 3 composite have isotropic permittivity values of about 4 and 6.5, respectively, the FGM exhibits two very different behaviors.
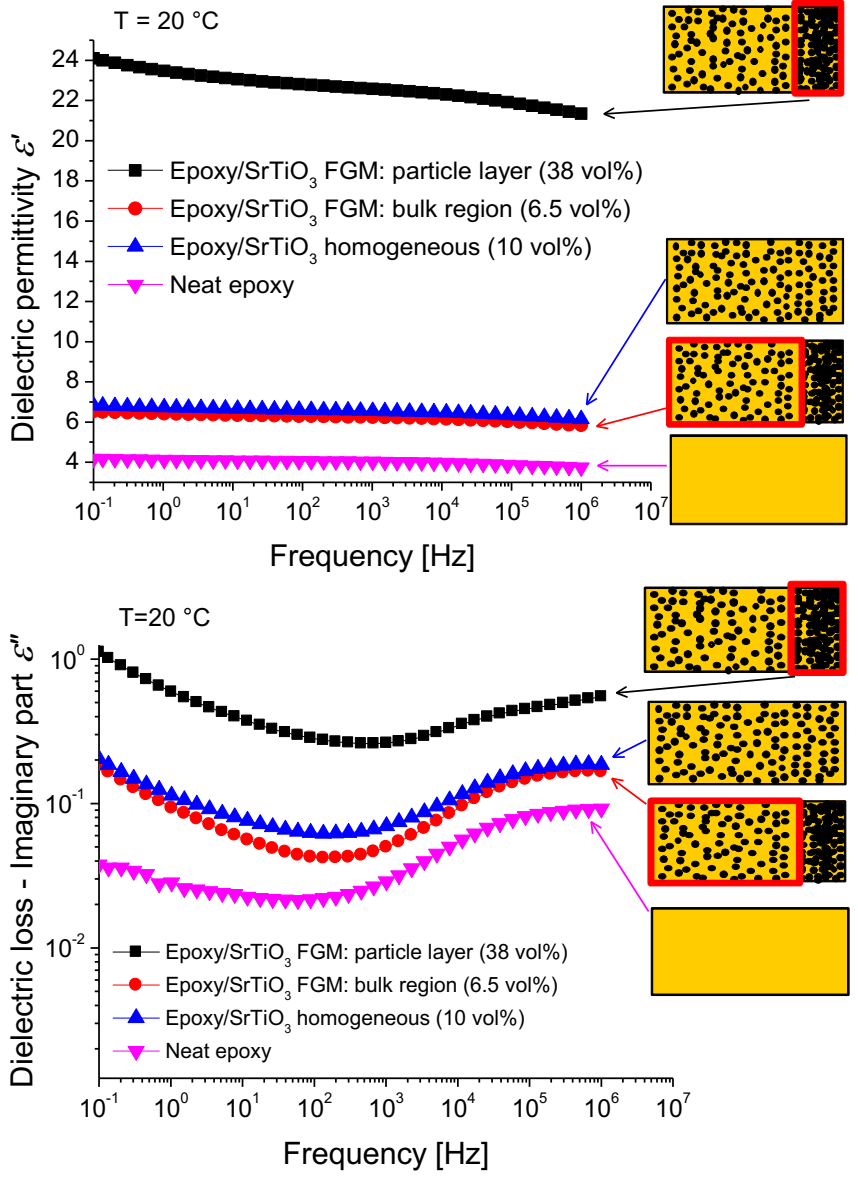

Figure 12. Complex dielectric permittivity versus frequency in the two regions (accumulated and depleted) in the epoxy/SrTiO 3 FGM composite processed at $250 \mathrm{~V} / \mathrm{mm}$ for 60 minutes. Comparison with the homogeneous epoxy $/ \mathrm{SrTiO}_{3}$ composite $(10 \mathrm{vol} \%)$ and the neat epoxy.

Firstly, one can observe that the permittivity and the dielectric loss of the depleted bulk region are almost similar with the ones of the homogeneous composite. Such properties are related to the fact that the particle content of the depleted bulk region is roughly unchanged during the electrophoretic process, as confirmed before. On the contrary, when this few vol\% of depleted particles is accumulated in the particle layer at the HV electrode, the particle concentration is largely increased. Consequently, the permittivity and loss of the accumulated layer is significantly increased at the $\mathrm{HV}$ electrode with values of the permittivity above 20 in these tested conditions and in the frequency range under consideration. The dielectric losses, even if slightly higher, are not controlled by a conduction process but mainly by a dipolar polarization process, as the low frequency slope is largely lower than -1 . 


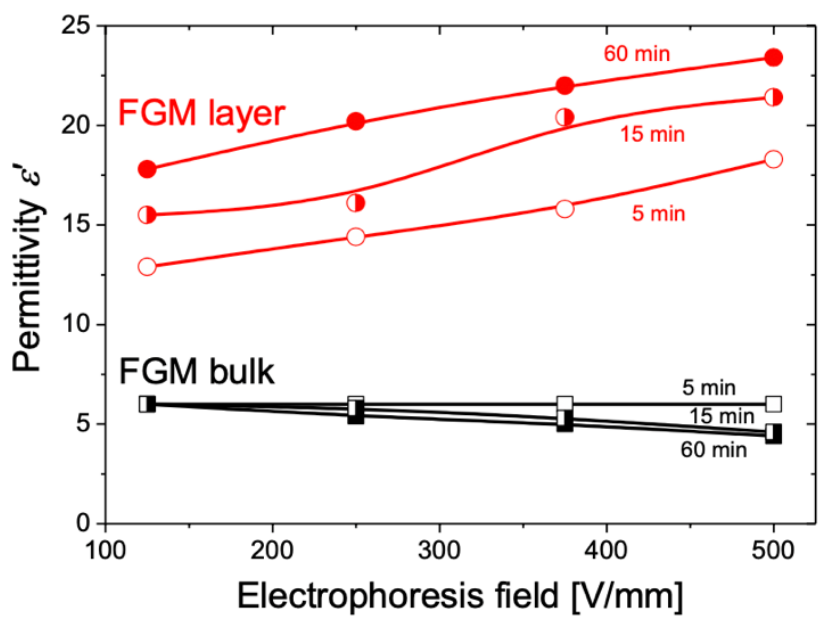

Figure 13. Dielectric permittivity in the two regions (accumulated and depleted) of various epoxy/ $\mathrm{SrTiO}_{3}$ FGM composites versus the electrophoresis field and for different times.

Finally, Figure 13 shows a summary of the permittivity changes in the two regions (accumulated and depleted) of initially $10 \mathrm{vol} \%$ epoxy $/ \mathrm{SrTiO}_{3} \mathrm{FGM}$ composites as a function of the electrophoresis process parameters (i.e. field and time). One can observe the possibility to tune the permittivity particularly in the particle layer region in a wide range of values in order to adapt it for a given application. Thus, the permittivity gradient of the entire FGM can be tailored to optimally minimize an electric field reinforcement region in the vicinity of the high voltage electrode. This principle can be easily extended to other physical properties applying the same methodology than the one developed here. In that purpose, other particle types could be used for the construction of property gradients by this electrophoretic method such as $\mathrm{BaTiO}_{3}, \mathrm{TiO}_{2}, \mathrm{ZnO}, \mathrm{SiC}$, etc. These particles have either high permittivity (ferroelectric) $>>100$ or nonlinear conduction effects. This new way of tailoring FGM composites paves the way of the possibility to adapt and relax the physical constraints in any system with multiple materials in contact each other's. This could participate to improve their reliability in extreme working conditions.

\section{CONCLUSION}

In this first paper, it was reported a novel way to build advanced functionally graded materials (FGM) based on polymer/ceramic composites using an electrophoretic deposition process. It relies on applying a DC voltage on the composite compound at the liquid state before curing it in order to 'freeze' the ceramic particles exactly where they have been accumulated. To exemplify this principle, a FGM epoxy/ $\mathrm{SrTiO}_{3}$ composite with a permittivity gradient is presented and the analysis methodology to build and characterize it is reported. An accumulated particle region is observed at the high voltage electrode with a field-dependent thickness, while the depleted bulk region remains almost unmodified. Moreover, the accumulated particle region exhibits a filler densification with the electrophoresis field and/or time, which enables the tuning of its permittivity. Such process can be very versatile to various electronic systems by directly using their own electrodes. This process is easily transferable and adaptable to a wide range of polymer/particle combinations to tailor their electrical conductivity, mechanical and thermal properties in a gradient form. This work paves the way to the development of more robust electronic systems using such FGM with properties gradient in order to relax the physical constraints in critical regions and to finally improve their overall reliability. The next Part 2 of this article series will present permittivity gradient features under a non-uniform electric field.

\section{ACKNOWLEDGMENT}

The authors would like to thank the French Research National Agency (ANR) for funding this work in the frame of the ELECTRON project N ${ }^{\circ}$ ANR-13-JS09-0007-01 (ANR JCJC 2013-2017).

\section{REFERENCES}

[1] S. Diaham, M.-L. Locatelli, and Z. Valdez-Nava, "Dielectrics for High Temperature SiC Device Insulation: Review of New Polymeric and Ceramic Materials," in Silicon Carbide - Materials, Proc. and Appl. in Electronics Devices, ed. M. Mukherjee, InTech, Rijeka, 2011, pp. 409-430.

[2] M. Sato et al, "Dynamic Potential Distributions of Surface Discharge in Silicone Gel," IEEE Trans. Dielec. Electr. Insul., vol. 22, pp. 1733$1738,2015$.

[3] X. Huang, P. Jiang, and T. Tanaka, "A Review of Dielectric Polymer Composites With High Thermal Conductivity," IEEE Electr. Insul. Mag., vol. 27, pp. 8-16, 2011.

[4] Z. Li, K. Okamoto, Y. Ohki, and T. Tanaka, "Effects of Nano-filler Addition on Partial Discharge Resistance and Dielectric Breakdown Strength of Micro- $\mathrm{Al}_{2} \mathrm{O}_{3} /$ Epoxy Composite," IEEE Trans. Dielec. Electr. Insul., vol. 17, pp. 653-661, 2010.

[5] I. Plesa et al, "Properties of Polymer Composites Used in HighVoltage Applications," Polymers, vol. 8, 173, 2016.

[6] M. Xiao and B.X. Du, "Review of High Thermal Conductivity Polymer Dielectrics for Electrical Insulation," High Volt, vol. 1, pp. 34-42, 2016.

[7] Z. Shen, X. Wang, T. Zhang, and Z. Jia, "In situ electric field driven assembly to construct adaptive graded permittivity $\mathrm{BaTiO}_{3} /$ epoxy resin composites for improved insulation performance", Applied Materials Today, vol. 20, No. 2020, pp. 100647, 2020.

[8] Z. Lin et al, "Exfoliated Hexagonal Boron Nitride-Based Polymer Nanocomposite with Enhanced Thermal Conductivity for Electronic Encapsulation," Comp. Sci. Technol., vol. 90, pp. 123-128, 2014.

[9] S. Diaham et al, "Huge Improvements of Electrical Conduction and Dielectric Breakdown in Polyimide/BN Nanocomposites," IEEE Trans. Dielec. Electr. Insul., vol. 23, pp. 2795-2803, 2016.

[10] D.D.L. Chung, "Composite materials for electrical applications," in Engineering Materials and Processes, ed. B. Derby, Springer-Verlag, London, 2003, pp. 73-89.

[11] D. Tan and P. Irwin, "Polymer Based Nanodielectric Composites", in Advance in Ceramics - Electric and Magnetic Ceramics, Bioceramics, Ceramics and Environment, ed. C. Sikalidis, InTech, Rijeka, 2011, pp. 115-132.

[12] L. Ramajo, M.M. Reboredo, and M.S. Castro, "BaTiO 3 -Epoxy Composites for Electronic Applications," Int. J. Appl. Ceram. Technol., vol. 7, pp. 444-451, 2010.

[13] J. Nunes-Pereira, C.M. Costa, and S. Lanceros-Méndez, "Polymer Composites and Blends for Battery Separators: State of the Art, Challenges and Future Trends," J. Power Sources, vol. 281, pp. 378$398,2015$. 
[14] A. Gupta, and M. Talha, "Recent Development in Modeling and Analysis of Functionally Graded Materials and Structures," Progr. Aeros. Sci., vol. 79, pp. 1-14, 2015.

[15] K. Kato et al, "Application of Functionally Graded Material for Solid Insulator in Gaseous Insulation System," IEEE Trans. Dielec. Electr. Insul., vol. 13, pp. 362-372, 2006.

[16] M. Kurimoto et al, "Application of Functionally Graded Material for Reducing Electric Field on Electrode and Spacer Interface," IEEE Trans. Dielec. Electr. Insul., vol. 17, pp. 256-263, 2010.

[17] N.J. Lee et al, "Characterization of Functionally Gradient Epoxy/Carbon Fiber Composite Prepared under Centrifugal Force," J. Mater. Sci., vol. 32, pp. 2013-2020, 1997.

[18] M. Krumova, C. Klingshirn, F. Haupert, and K. Friedrich, "Microhardness Studies on Functionally Graded Polymer Composites," Comp. Sci. Technol., vol. 61, pp. 557-563, 2001.

[19] L. Besra and M. Liu, "A Review on Fundamentals and Applications of Electrophoretic Deposition (EPD)," Progr. Mater. Sci., vol. 52, pp. 1-61, 2007.

[20] S. Put, J. Vleugels and O. Van Der Biest, "Gradient Profile Prediction in Functionally Graded Materials Processed by Electrophoretic Deposition," Acta Mater., vol. 51, 6303-6317, 2003.

[21] S. Diaham et al, "Method for processing an electrically insulating material providing same with self-adjusting electric field grading properties for electrical components", International Patent, WO2017211847A1, 14 December, 2017; PCT/EP2017/063740, 6 June 2017; FR1655173, 6 June 2016.

[22] S. Diaham et al, "An Original Way to Tailor Field Grading Composite Materials with Permittivity Gradient by Electrophoresis," IEEE Int. Conf. Dielectr. (ICD), pp. 1-4, 2018.

[23] L. Lévêque, "Nouveaux matériaux composites à gradient de permittivité structurés par un champ électrique et leur application pour la gradation de potentiel,hD dissertation," University of Toulouse, 2017 (in French).

[24] J. Zhang, and B. Lee, "Electrophoretic Deposition and Characterization of Micrometer-Scale $\mathrm{BaTiO}_{3}$ Based X7R Dielectric Thick Films," J. Am. Ceram. Soc., vol. 83, pp. 2417-2422, 2000.

[25] P. Sarkar and P.S. Nicholson, "Electrophoretic Deposition (EPD): Mechanisms, Kinetics and Application to Ceramics," J. Am. Ceram. Soc., vol. 79, pp. 1987-2002, 1996.

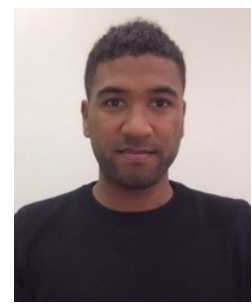

Sombel Diaham (M'16) was born in 1982 in Montauban, France. He was M.Sc. graduated in 2005 and received his PhD degree in 2007 in Electrical Engineering from University of Toulouse, France. In 2008, he joined the LAPLACE as Associate Professor. He received his Research Supervision Habilitation (HDR) from University of Toulouse in 2017. His research work deals with insulating polymers for high voltage power electronics.

Since 2009, he develops a research activity on composites (nanodielectrics and (di)electrophoretic-structured composites) for power electronics. Since 2018, he has been an invited researcher in Analog Devices, Limerick (Ireland), working on insulation design for HV digital isolators used in isolated-gate drivers. He co-authored 42 papers in international scientific journals, 68 papers presented in international conferences, 5 book chapters, 1 book as Guest Editor and 6 granted patents.

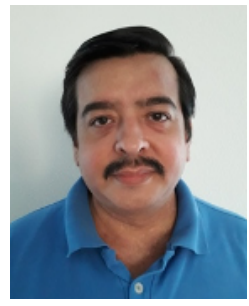

Zarel Valdez-Nava (M'16). He received his $\mathrm{PhD}$ in Materials Science in 2005 jointly from University of Toulouse, France and the Autonomous University of Nuevo Leon, Mexico. In 2008, he joined the LAPLACE as Researcher of the French National Scientific Research Center (CNRS). His research work deals with insulating and dielectric materials for high voltage and/or for high temperature power electronics.
His interests concern the relationship between the processing of composite and ceramic materials in regards of their performance in real electrical engineering applications. He has co-authored over 30 papers in international scientific journals.

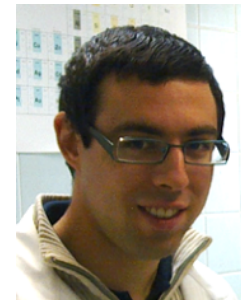

Louis Lévêque was born in 1989 in France. He received his Engineering degree in Materials Science from the University of Nantes (Polytech Nantes) in 2012, and his PhD degree in Electrical Engineering from the University of Toulouse in 2017. He is contributed as $\mathrm{PhD}$ student in the framework of the ELECTRONANR project. From 2017-2019, he worked on the PROCETS European project at Tenneco, Belgium.

In 2020, he joined the High School Saint-Exupéry in Bellegarde-SurValserine (Valserhône, France), where he is a teacher of Science and Technology in Industry and Sustainable Development.

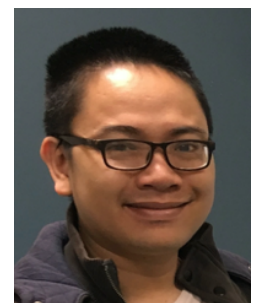

Trung Trong Le was born in 1986 in Thanh Hoa, Vietnam. He obtained his MSc degree in Electrical Engineering from Hanoi University of Science and Technology in 2009 and received the $\mathrm{PhD}$ degree from the University of Toulouse in 2016. He contributed as a postdoctoral researcher in the framework of the ELECTRON-ANR project. He is currently a research associate at Laplace Laboratory and research engineer at IRT Saint-Exupéry, France, working on application of FGM composites to power electronic modules.

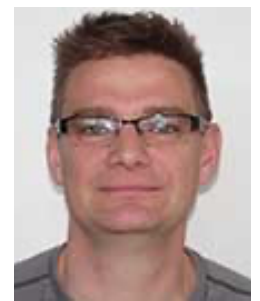

Lionel Laudebat was born in Toulouse, France, in 1974. Former student of the Brittany site of the Ecole Normale Supérieur de Cachan. $\mathrm{He}$ received the "Agrégation" in Electrical Engineering in 1998 and the $\mathrm{PhD}$ degree from the University of Toulouse III in 2003. He is currently an Associate Professor of Electrical Engineering at the University of Toulouse, Albi site (France).

He is associated researcher in LAPLACE since 2015. His main research interests include numerical simulation and modeling of power electronic materials, studies of dielectric materials included modeling and characterization especially for ceramics and composite materials.

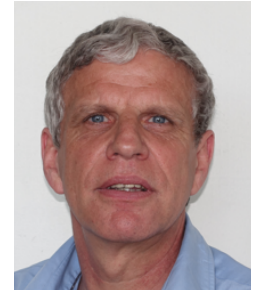

Thierry Lebey (M'98) received his M.Sc. degree in Solid State Physics in 1984, his Ph.D. in Electrical Engineering in 1989, both from University Paul Sabatier in Toulouse. Since 1990, he is engaged with the French National Scientific Research Center (CNRS) where he is now Senior Research Scientist. He is the author of more than 80 journal and 130 conference papers and holds 10 international patents.

From 2016 to 2019, he was Director of the Laboratory of Plasma and Energy Conversion - LAPLACE - in Toulouse. Since 2020, he is working as an high voltage expert for Safran Group. 